\title{
Kasner Universe in Creation-Field Cosmology
}

\author{
Kishor S. Adhav, Meena V. Dawande, Maya S. Desale, Ranjita B. Raut \\ Department of Mathematics, Sant Gadge Baba Amravati University, Amravati, India \\ E-mail: atiksadhav@yahoo.co.in \\ Received April 18, 2010; revised July 9, 2010; accepted July 20, 2010
}

\begin{abstract}
We have studied the Hoyle-Narlikar $C$-field cosmology with Kasner [1,2] space-time. Using methods of Narlikar and Padmanabhan [3], the solutions have been studied when the creation field $C$ is a function of time $t$ only. The geometrical and physical properties of the models, thus obtained, are also studied.
\end{abstract}

Keywords: Kasner Space-Time, Creation Field Cosmology, Cosmological Models of Universe.

\section{Introduction}

The three important observations in astronomy viz., the phenomenon of expanding universe, primordial nucleonsynthesis and the observed isotropy of cosmic microwave background radiation (CMBR) were supposed to be successfully explained by big-bang cosmology which is based on Einstein's field equations. However, Smoot et al. [4] revealed that the earlier predictions of the Friedman-Robertson-Walker types of models do not always exactly meet our expectations. Some puzzling results regarding the red shifts from the extra galactic objects continue to contradict the theoretical explanations given from the big bang type of the models. Also, CMBR discovery did not prove it to be an out come of big bang theory. Infact, Narlikar et al. [5] proved the possibility of non-relic interpretation of CMBR. To explain such phenomenon, many alternative theories have been proposed from time to time. Hoyle [6], Bondi and Gold [7] proposed steady state theory in which the universe does not have singular beginning nor an end on the cosmic time scale. Moreover, they have shown that the statistical properties of the large scale features of the universe do not change. Further, the constancy of the mass density has been accounted by continuous creation of matter going on in contrast to the one time infinite and explosive creation of matter at $t=0$ as in the earlier standard models. But, the principle of conservation of matter was violated in this formalism. To overcome this difficulty, Hoyle and Narlikar [8] adopted a field theoretic approach by introducing a massless and chargeless scalar field $C$ in the Einstein-Hilbert action to account for the matter creation. In the $C$-field theory introduced by Hoyle and Narlikar there is no big bag type of singularity as in the steady state theory of Bondi and Gold [7].
The solutions of Einstein's field equations admitting radiation with negative energy mass less scalar creation field $C$ was obtained by Narlikar and Padmanabhan [3]. The study of Hoyle and Narlikar theory $[9,10]$ and [8] to the space-time of dimensions more than four was carried out by Chatterjee and Banerjee [11]. RajBali and Tikekar [12] studied $C$-field cosmology with variable $G$ in the flat Friedmann-Robertson-Walker model. Whereas, $C$-field cosmological models with variable $G$ in FRW space-time has been studied by RajBali and Kumawat [13]. Singh and Chaubey [14] studied various Bianchi types models and Kantowski-Sach model in creation field cosmology.

The way in which the Kasner [1,2] metric has played a central role in the elucidation of the existence and structure of anisotropic cosmological models and their singularities in general relativity motivates the authors to study this problem. The Kasner metric is one of the more widely studied metric. Its usefulness for the construction of cosmological models and its utility for certain studies of elementary particles have made it particularly attractive for exploitation. Because of its simplicity it has been "rediscovered" many times and is itself very closely related to metrics given several years earlier by Weyl, Levi-Civita and Wilson. The form in which Kasner presented it has been virtually forgotten in favor of the dynamic form of the synchronous Bianchi I metric. Here, we have considered a spatially homogeneous and anisotropic Kasner form of Bianchi type-I metric in Hoyle and Narlikar $C$-field cosmology. We have assumed that the creation field $C$ is a function of time $t$ only i.e. $C(x, t)$ $=C(t) \cdot$

\section{Hoyle and Narlikar C-Field Cosmology}

Introducing a massless scalar field called as creation 
field viz. $C$-field, Einstein's field equations are modified. Einstein's field equations modified by Hoyle and Narlikar [8-10] are

$$
R_{i j}-\frac{1}{2} g_{i j} R=-8 \pi\left({ }^{m} T_{i j}+{ }^{c} T_{i j}\right)
$$

where ${ }^{m} T_{i j}$ is matter tensor of Einstein theory and ${ }^{c} T_{i j}$ is matter tensor due to the $C$-field which is given by

$$
{ }^{c} T_{i j}=-f\left(C_{i} C_{j}-\frac{1}{2} g_{i j} C^{k} C_{k}\right)
$$

where $f>0$ and $C_{i}=\frac{\partial C}{\partial x^{i}}$.

Because of the negative value of $T^{00}\left(T^{00}<0\right)$, the $C$-field has negative energy density producing repulsive gravitational field which causes the expansion of the universe. Hence, the energy conservation equation reduces to

$$
{ }^{m} T_{; j}^{i j}=-{ }^{c} T_{; j}^{i j}=f C^{i} C^{j}{ }_{; j}
$$

i.e. matter creation through non-zero left hand side is possible while conserving the over all energy and momentum.

[Here semicolon (;) denotes covariant derivative]. Above equation is similar to

$$
m g_{i j} \frac{d x^{i}}{d s}-C_{j}=0
$$

Which implies that the 4-momentum of the created particle is compensated by the 4-momentum of the $C$-field. In order to maintain the balance, the $C$-field must have negative energy. Further, the $C$-field satisfy the source equation $f C_{; i}^{i}=J_{; i}^{i}$ and $J^{i}=\rho \frac{d x^{i}}{d s}=\rho v^{i}$, where $\rho$ is homogeneous mass density.

\section{Metric and Field Equations}

We consider an anisotropic [Bianchi type-I] metric in Kasner form as

$$
d s^{2}=d t-t^{2 q_{1}} d x^{2}-t^{2 q_{2}} d y^{2}-t^{2 q_{3}} d z^{2}
$$

where $q_{1}, q_{2}$ and $q_{3}$ are three parameters that we shall require to be constants.

We have assumed that creation field $C$ is function of time $t$ only i.e. $C(x, t)=C(t)$ and

$$
{ }^{m} T_{j}^{i}=\operatorname{diag}(\rho,-p,-p,-p)
$$

We have also assumed that velocity of light and gravitational constant are equal to one unit.

We first find the components of Ricci tensor $R_{i j}$.

Assuming the metric (5), the non vanishing components of Christoffel's symbols are

$$
\Gamma_{i i}^{0}=q_{i} t^{2 q_{i}-1}, \quad \Gamma_{i 0}^{i}=\frac{q_{i}}{t}, i=1,2,3 .
$$

Hence, we calculate

$$
\begin{aligned}
& R_{i i}=-q_{i}\left(q_{1}+q_{2}+q_{3}-1\right) t^{2 q_{i}-2}, i=1,2,3 . \\
& R_{00}=-\left[\left(q_{1}+q_{2}+q_{3}\right)-\left(q_{1}^{2}+q_{2}^{2}+q_{3}^{2}\right)\right] t^{-2} .
\end{aligned}
$$

Let $S=q_{1}+q_{2}+q_{3}$ and $\theta=q_{1}^{2}+q_{2}^{2}+q_{3}^{2}$, we get $R=\left(S^{2}-2 S+\theta\right) t^{-2}$

Now, the Hoyle-Narlikar field Equations (1) for metric (5) with the help of Equations (2), (3) and (6) can be written as

$$
\begin{aligned}
& {\left[q_{1}(S-1)-\frac{1}{2}\left(S^{2}-2 S+\theta\right)\right] t^{-2}=8 \pi\left(p-\frac{1}{2} f \dot{C}^{2}\right)} \\
& {\left[q_{2}(S-1)-\frac{1}{2}\left(S^{2}-2 S+\theta\right)\right] t^{-2}=8 \pi\left(p-\frac{1}{2} f \dot{C}^{2}\right)} \\
& {\left[q_{3}(S-1)-\frac{1}{2}\left(S^{2}-2 S+\theta\right)\right] t^{-2}=8 \pi\left(p-\frac{1}{2} f \dot{C}^{2}\right)} \\
& {\left[(S-\theta)+\frac{1}{2}\left(S^{2}-2 S+\theta\right)\right] t^{-2}=8 \pi\left(\rho-\frac{1}{2} f \dot{C}^{2}\right)} \\
& \dot{\rho}+\frac{S}{t}(\rho+p)=f \dot{C}\left(\ddot{C}+\dot{C} \frac{S}{t}\right)
\end{aligned}
$$

Now, we assume that

$$
V=t^{\left(q_{1}+q_{2}+q_{3}\right)}=t^{S}
$$

Above Equation (12) can be written in the form

$$
\frac{d}{d V}(V \rho)+p=f \dot{C}(V) \frac{d}{d V}[V \dot{C}(V)]
$$

In order to obtain a unique solution, one has to satisfy the rate of creation of matter-energy (at the expense of the negative energy of the $C$-field).

Without loss of generality, we assume that the rate of creation of matter energy density is proportional to the strength of the existing $C$-field energy-density per unit proper-volume.

This is given by

$$
\frac{d}{d V}(V \rho)+p=\alpha^{2} \dot{C}^{2} \equiv \alpha^{2} g^{2}(V)
$$

where $\alpha$ is proportionality constant .

Let us define that $\dot{C}(V) \equiv g(V)$.

Substituting it in (13), we get

$$
\frac{d}{d V}(V \rho)+p=f g(V) \frac{d}{d V}(V g)
$$

Comparing right hand sides of (13) and (14), we get 


$$
g(V) \frac{d}{d V}(g V)=\frac{\alpha^{2}}{f} g^{2}(V) .
$$

On integrating which gives

$$
g(V)=A_{1} V^{\left(\frac{\alpha^{2}}{f}-1\right)}
$$

where $A_{1}$ is arbitrary constant of integration.

We consider the equation of state of matter as

$$
p=\gamma \rho, 0 \leq \gamma \leq 1
$$

Substituting Equations (17) and (18) in the equation (14), we get

$$
\frac{d}{d V}(V \rho)+\gamma \rho=\alpha^{2} A^{2} V^{2\left(\frac{\alpha^{2}}{f}-1\right)}
$$

which further yields

$$
\rho=\frac{\alpha^{2} A_{1}^{2}}{\left(2 \frac{\alpha^{2}}{f}-1+\gamma\right)} V^{2\left(\frac{\alpha^{2}}{f}-1\right)}
$$

Subtracting Equation (7) from Equation (8), we get

$$
\left(q_{2}-q_{1}\right)(S-1) t^{-2}=0
$$

Equation (21) can be written as

$$
\frac{d}{d t}\left(\frac{q_{1}}{t}-\frac{q_{2}}{t}\right)+\left(\frac{q_{1}}{t}-\frac{q_{2}}{t}\right) \frac{S}{t}=0
$$

Now, using Equation (12), the above equation becomes

$$
\frac{d}{d t}\left(\frac{q_{1}}{t}-\frac{q_{2}}{t}\right)+\left(\frac{q_{1}}{t}-\frac{q_{2}}{t}\right) \frac{\dot{V}}{V}=0
$$

This on integration gives

$$
\frac{t^{q_{1}}}{t^{q_{2}}}=d_{1} e^{x_{1} \int \frac{d t}{V}}, d_{1}=\text { const } ; \quad x_{1}=\text { const }
$$

Subtracting Equation (9) from Equation (8), we get

$$
\left(q_{2}-q_{3}\right)(S-1) t^{-2}=0
$$

Equation (24) can be written as

$$
\frac{d}{d t}\left(\frac{q_{2}}{t}-\frac{q_{3}}{t}\right)+\left(\frac{q_{2}}{t}-\frac{q_{3}}{t}\right) \frac{S}{t}=0
$$

Now, using Equations (12) the above equation becomes

$$
\frac{d}{d t}\left(\frac{q_{2}}{t}-\frac{q_{3}}{t}\right)+\left(\frac{q_{2}}{t}-\frac{q_{3}}{t}\right) \frac{\dot{V}}{V}=0 .
$$

This on integration gives

$$
\frac{t^{q_{2}}}{t^{q_{3}}}=d_{2} e^{x_{2} \int \frac{d t}{V}} ; d_{2}=\text { const } ; \quad x_{2}=\text { const }
$$

Subtracting Equation (9) from Equation (7), we get

$$
\left(q_{1}-q_{3}\right)(S-1) t^{-2}=0
$$

Equation (26) can be written as

$$
\frac{d}{d t}\left(\frac{q_{1}}{t}-\frac{q_{3}}{t}\right)+\left(\frac{q_{1}}{t}-\frac{q_{3}}{t}\right) \frac{S}{t}=0
$$

Now, using Equations (12) the above equation becomes

$$
\frac{d}{d t}\left(\frac{q_{1}}{t}-\frac{q_{3}}{t}\right)+\left(\frac{q_{1}}{t}-\frac{q_{3}}{t}\right) \frac{\dot{V}}{V}=0
$$

This on integration gives

$$
\frac{t^{q_{1}}}{t^{q_{3}}}=d_{3} e^{x_{3} \int \frac{d t}{V}} \quad d_{3}=\text { const } ; \quad x_{3}=\text { const }
$$

where $d_{3}=d_{1} d_{2} ; x_{3}=x_{1}+x_{2}$.

Using Equations (23), (25) and (27), the values of $t^{q_{1}}, t^{q_{2}}$ and $t^{q_{3}}$ can be explicitly written as,

$$
\begin{aligned}
& t^{q_{1}}=D_{1} V^{1 / 3} \exp \left(X_{1} \int \frac{d t}{V}\right) \\
& t^{q_{2}}=D_{2} V^{1 / 3} \exp \left(X_{2} \int \frac{d t}{V}\right) \\
& t^{q_{3}}=D_{3} V^{1 / 3} \exp \left(X_{3} \int \frac{d t}{V}\right)
\end{aligned}
$$

where the relations $D_{1} D_{2} D_{3}=1$ and $X_{1}+X_{2}+X_{3}=0$ are satisfied by $D_{i}(i=1,2,3)$ and $X_{i}(i=1,2,3)$.

Adding Equations (7)-(9) and subtracting from three times Equation (10), we get

$$
S(S-1) t^{-2}=12 \pi(\rho-p)
$$

From Equation (12) and (18), we get

$$
\frac{\ddot{V}}{V}=12 \pi(1-\gamma) \rho
$$

Substituting Equation (21) in Equation (32), we get

$$
\frac{\ddot{V}}{V}=\frac{12 \pi(1-\gamma) \alpha^{2} A_{1}^{2}}{\left(2 \frac{\alpha^{2}}{f}-1+\gamma\right)} V^{2\left(\frac{\alpha^{2}}{f}-1\right)}
$$

This further gives

$$
V=\left\{A_{1}\left(f-\alpha^{2}\right)\left[\frac{12 \pi(1-\gamma)}{\left(2 \alpha^{2}-f+\gamma f\right)}\right]^{1 / 2}\right\}^{\frac{f}{f-\alpha^{2}}} \frac{f}{t^{f-\alpha^{2}}}
$$

Substituting Equation (34) in Equation (17), we get

$$
g=\frac{1}{\left(f-\alpha^{2}\right)}\left[\frac{12 \pi(1-\gamma)}{\left(2 \alpha^{2}-f+\gamma f\right)}\right]^{-1 / 2} \frac{1}{t}
$$

Also, from equation $\dot{C}(V)=g(V)$, we get 


$$
C=\frac{1}{\left(f-\alpha^{2}\right)}\left[\frac{12 \pi(1-\gamma)}{\left(2 \alpha^{2}-f+\gamma f\right)}\right]^{-1 / 2} \log t
$$

Substituting Equation (34) in Equation (21), the homogeneous mass density becomes

$$
\rho=\frac{\alpha^{2} f}{12 \pi(1-\gamma)\left(f-\alpha^{2}\right)^{2}} \frac{1}{t^{2}}
$$

Using Equation (18), pressure becomes

$$
p=\frac{\alpha^{2} f \gamma}{12 \pi(1-\gamma)\left(f-\alpha^{2}\right)^{2}} \frac{1}{t^{2}}
$$

From Equations (37) and (38), it is observed that:

When time $t \rightarrow \infty$, we get, density and pressure tending to zero i.e. the model reduces to vacuum. Also from Equation (31), we can verify that $[p=0$ and $\rho=0$ gives] $S=1$. Which is consistent with the Kasner's condition for vacuum i.e. $\mathrm{S} \equiv q_{1}+q_{2}+q_{3}=1$.

When $f=\alpha^{2}$, there is singularity in density and pressure.

There is also singularity in density and pressure for $\gamma=1$.

From Equation (18), for $\gamma=1$, we get $p=\rho$ which further gives [using Equation (31)] $S=1$. In this case, we can interpret this result as "an anisotropic Kasner type universe can be considered to be filled with an ideal (non-viscous) fluid which has equation of state $p=\rho$ [stiff matter: the velocity of sound coincides with the speed of light].

Using Equation (34) in Equations (28)-(30) we get

$$
\begin{aligned}
t^{q_{1}} & =D_{1} K^{1 / 3} t^{\frac{f}{3\left(f-\alpha^{2}\right)}} \exp \left[\frac{X_{1}}{K}\left(1-\frac{f}{\alpha^{2}}\right) t^{\frac{\alpha^{2}}{\alpha^{2}-f}}\right] \\
t^{q_{2}} & =D_{2} K^{1 / 3} t^{\frac{f}{3\left(f-\alpha^{2}\right)}} \exp \left[\frac{X_{2}}{K}\left(1-\frac{f}{\alpha^{2}}\right) t^{\frac{\alpha^{2}}{\alpha^{2}-f}}\right] \\
t^{q_{3}} & =D_{3} K^{1 / 3} t^{\frac{f}{3\left(f-\alpha^{2}\right)}} \exp \left[\frac{X_{3}}{K}\left(1-\frac{f}{\alpha^{2}}\right) t^{\frac{\alpha^{2}}{\alpha^{2}-f}}\right]
\end{aligned}
$$

where $K=\left\{A_{1}\left(f-\alpha^{2}\right)\left[\frac{12 \pi(1-\gamma)}{\left(2 \alpha^{2}-f+\gamma f\right)}\right]^{1 / 2}\right\}^{\frac{f}{f-\alpha^{2}}}$ and $D_{1}, D_{2}, D_{3}$ and $X_{1}, X_{2}, X_{3}$ are constants of integration, satisfying the relations $D_{1} D_{2} D_{3}=1$ and $X_{1}+X_{2}+X_{3}=0$.

\section{Physical Properties}

We define $a=\left(t^{q_{1}} t^{q_{2}} t^{q_{3}}\right)^{\frac{1}{3}}$ as the average scale factor so that the Hubble's parameter in our anisotropic models may be defined as

$H=\frac{\dot{a}}{a}=\frac{1}{3} \sum_{i=1}^{3} H_{i}$, where $H_{i}=\frac{\dot{a}_{i}}{a_{i}}$ are directional

Hubble's factors in the direction of $x^{i}$ s respectively.

The expansion scalar $\theta$ is given by

$$
\theta=3 H=\left(\frac{f}{f-\alpha^{2}}\right) \frac{1}{t}
$$

The mean anisotropy parameter is given by

$$
\begin{gathered}
A=\frac{1}{3} \sum_{i=1}^{3}\left(\frac{\Delta H_{i}}{H}\right)^{2} \\
A=\frac{3 X^{2}}{K^{2}}\left(\frac{f-\alpha^{2}}{f}\right)^{2} t^{2\left(\frac{\alpha^{2}}{\alpha^{2}-f}\right)}
\end{gathered}
$$

The shear scalar $\sigma^{2}$ is given by

$$
\begin{gathered}
\sigma^{2}=\frac{1}{2}\left(\sum_{i=1}^{3} H_{i}^{2}-3 H^{2}\right)=\frac{3}{2} A H^{2} \\
=\frac{X^{2}}{2 K^{2}} t^{2\left(\frac{f}{\alpha^{2}-f}\right)}
\end{gathered}
$$

The deceleration parameter is given by

$$
\begin{gathered}
q=\frac{d}{d t}\left(\frac{1}{H}\right)-1 \\
q=2-\frac{3 \alpha^{2}}{f},
\end{gathered}
$$

where $X^{2}=X_{1}^{2}+X_{2}^{2}+X_{3}^{2}$

If $f>\alpha^{2}$ then for large $t$, the model tends to isotropic case.

Case I : $\gamma=0$ (Dust Universe) :

In this case, we obtain the values of various parameters as

$$
\begin{gathered}
g=\frac{1}{f-\alpha^{2}}\left[\frac{\left(2 \alpha^{2}-f\right)}{12 \pi}\right]^{1 / 2} \frac{1}{t} \\
C=\frac{1}{f-\alpha^{2}}\left[\frac{\left(2 \alpha^{2}-f\right)}{12 \pi}\right]^{1 / 2} \log t \\
\rho=\frac{\alpha^{2} f}{12 \pi\left(f-\alpha^{2}\right)^{2}} \frac{1}{t^{2}},
\end{gathered}
$$




$$
\begin{aligned}
& t^{q_{1}}=D_{1} K_{1}^{1 / 3} t^{\frac{f}{3\left(f-\alpha^{2}\right)}} \exp \left[\frac{X_{1}}{K_{1}}\left(1-\frac{f}{\alpha^{2}}\right) t^{\frac{\alpha^{2}}{\alpha^{2}-f}}\right] \\
& t^{q_{2}}=D_{2} K_{1}^{1 / 3} t^{\frac{f}{3\left(f-\alpha^{2}\right)}} \exp \left[\frac{X_{2}}{K_{1}}\left(1-\frac{f}{\alpha^{2}}\right) t^{\frac{\alpha^{2}}{\alpha^{2}-f}}\right] \\
& t^{q_{3}}=D_{3} K_{1}^{1 / 3} t^{\frac{f}{3\left(f-\alpha^{2}\right)}} \exp \left[\frac{X_{3}}{K_{1}}\left(1-\frac{f}{\alpha^{2}}\right) t^{\frac{\alpha^{2}}{\alpha^{2}-f}}\right]
\end{aligned}
$$

where $K_{1}=\left\{A\left(f-\alpha^{2}\right)\left[\frac{12 \pi}{\left(2 \alpha^{2}-f\right)}\right]^{1 / 2}\right\}^{\frac{f}{f-\alpha^{2}}}$

Here $D_{1}, D_{2}, D_{3}$ and $X_{1}, X_{2}, X_{3}$ are constants of integration, satisfying the relations $D_{1} D_{2} D_{3}=1$ and $X_{1}+X_{2}+X_{3}=0$.

In this case, the expansion scalar $\theta$ is given by

$$
\theta=\left(\frac{f}{f-\alpha^{2}}\right) \frac{1}{t}
$$

The mean anisotropy parameter is given by

$$
A=\frac{3 X^{2}}{K_{1}^{2}}\left(\frac{f-\alpha^{2}}{f}\right)^{2} t^{2\left(\frac{\alpha^{2}}{\alpha^{2}-f}\right)}
$$

The shear scalar $\sigma^{2}$ is given by

$$
\sigma^{2}=\frac{X^{2}}{2 K_{1}^{2}} t^{2\left(\frac{f}{\alpha^{2}-f}\right)}
$$

The deceleration parameter is given by

$$
q=2-\frac{3 \alpha^{2}}{f}
$$

where $X^{2}=X_{1}^{2}+X_{2}^{2}+X_{3}^{2}$

If $f>\alpha^{2}$, this model also tends to isotropy for large $t$.

\section{Case II : $\gamma=\frac{1}{3}$ (Disordered Radiation Universe)}

In this case, we obtain the values of various parameters as

$$
\begin{gathered}
g=\frac{1}{f-\alpha^{2}}\left[\frac{\left(3 \alpha^{2}-f\right)}{12 \pi}\right]^{1 / 2} \frac{1}{t} \\
C=\frac{1}{f-\alpha^{2}}\left[\frac{\left(3 \alpha^{2}-f\right)}{12 \pi}\right]^{1 / 2} \log t \\
\rho=\frac{3 \alpha^{2} f}{24 \pi\left(f-\alpha^{2}\right)^{2}} \frac{1}{t^{2}}, \\
p=\frac{\alpha^{2} f}{24 \pi\left(f-\alpha^{2}\right)^{2}} \frac{1}{t^{2}}
\end{gathered}
$$

$$
\begin{array}{r}
t^{q_{1}}=D_{1} K_{2}^{1 / 3} t^{\frac{f}{3\left(f-\alpha^{2}\right)}} \exp \left[\frac{X_{1}}{K_{2}}\left(1-\frac{f}{\alpha^{2}}\right) t^{\frac{\alpha^{2}}{\alpha^{2}-f}}\right] \\
t^{q_{2}}=D_{2} K_{2}^{1 / 3} t^{\frac{f}{3\left(f-\alpha^{2}\right)}} \exp \left[\frac{X_{2}}{K_{2}}\left(1-\frac{f}{\alpha^{2}}\right) t^{\frac{\alpha^{2}}{\alpha^{2}-f}}\right] \\
t^{q_{3}}=D_{3} K_{2}^{1 / 3} t^{\frac{f}{3\left(f-\alpha^{2}\right)}} \exp \left[\frac{X_{3}}{K_{2}}\left(1-\frac{f}{\alpha^{2}}\right) t^{\frac{\alpha^{2}}{\alpha^{2}-f}}\right]
\end{array}
$$

where $K_{2}=\left\{A_{1}\left(f-\alpha^{2}\right)\left[\frac{12 \pi}{\left(\alpha^{2}-f\right)}\right]^{1 / 2}\right\}^{\frac{f}{f-\alpha^{2}}}$.

Here $D_{1}, D_{2}, D_{3}$ and $X_{1}, X_{2}, X_{3}$ are constants of integration, satisfying the relations $D_{1} D_{2} D_{3}=1$ and $X_{1}+X_{2}+X_{3}=0$.

In this case, the expansion scalar $\theta$ is given by

$$
\theta=\left(\frac{f}{f-\alpha^{2}}\right) \frac{1}{t} .
$$

The mean anisotropy parameter is given by

$$
A=\frac{3 X^{2}}{K_{2}^{2}}\left(\frac{f-\alpha^{2}}{f}\right)^{2} t^{2\left(\frac{\alpha^{2}}{\alpha^{2}-f}\right)}
$$

The shear scalar $\sigma^{2}$ is given by

$$
\sigma^{2}=\frac{X^{2}}{2 K_{2}^{2}} t^{2\left(\frac{f}{\alpha^{2}-f}\right)}
$$

The deceleration parameter is given by

$$
q=2-\frac{3 \alpha^{2}}{f}
$$

where $X^{2}=X_{1}^{2}+X_{2}^{2}+X_{3}^{2}$ or $f>\alpha^{2}$, this model also tends to isotropy for large $t$.

\section{Discussion}

1) In both cases for $f>\alpha^{2}$, we get negative deceleration parameter indicating that the universe is accelerating. This observation is consistent with the present day observation.

2) The expansion scalar $\theta$ starts with an infinite value at $t=0$, further gradually decreases $\&$ the expansion halts when $t=\infty$.

3) For $f>\alpha^{2}$, we get $\lim _{t \rightarrow \infty} \frac{\sigma}{\theta} \neq 0$. Therefore, the models are isotropic for large value of $t$.

4) Also, we have noticed that matter density is inversely proportional to square of time $t$. When $t \rightarrow 0$, we 
get $\rho \rightarrow \infty$ and when $t \rightarrow \infty$, we get $\rho \rightarrow 0$.

These are all physically valid results indicating that there is a situation where Kasner type $C$-field cosmology starts from infinite mass density.

5) In general, we have observed that the creation field $C$ is proportional to time $t$. That is, the creation of matter increases as time increases.

\section{References}

[1] E. Kasner, "Geometrical Theorem on Einstein's Cosmological Equations," American Journal of Mathematics, Vol. 43, No. 2, 1921, pp. 217-221.

[2] E. Kasner, "An Algebraic Solution of the Einstein Equations," Transactions of the American Mathematical Society, Vol. 27, No. 1, 1925, pp. 101-105.

[3] J. V. Narlikar and T. Padmanabhan, "Creation-Field Cosmology: A Possible Solution to Singularity, Horizon, and Flatness Problems," Physical Review D, Vol. 32, No. 8, 1985, pp. 1928-1934.

[4] G. F. Smoot, et al., "Structure in the COBE Differential Microwave Radiometer First-Year Maps," Astrophysical Journal, Vol. 396, No. 1, 1992, pp. 21-25.

[5] J. V. Narlikar, et al., "Inhomogeneities in the Microwave Background Radiation Interpreted within the Framework of the Quasi-Steady State Cosmology," Astrphysical Journal, Vol. 585, No. 3, 2003, pp. 1-11.

[6] F. Hoyle, "A New Model for the Expanding Universe," Monthly Notices of the Royal Astronomical Society, Vol. 108, No. 1748, 1948, pp. 372-382.
[7] H. Bondi and T. Gold, "The Steady-State Theory of the Expanding Universe," Monthly Notices of the Royal Astronomical Society, Vol. 108, No. 3, 1948, pp. 252-270.

[8] F. Hoyle and J. V. Narlikar, "A Radical Departure from the 'Steady-State' Concept in Cosmology," Proeedings of Royal Society (London) A, Vol. 290, No. 1421, 1966, pp. 162-176.

[9] F. Hoyle and J. V. Narlikar, "Mach's Principle and the Creation of Matter," Proceedings of Royal Society (London) A, Vol. 273, No. 1352, 1963, pp. 1-11.

[10] F. Hoyle and J. V. Narlikar "The C-Field as a Direct Particle Field," Proceedings of Royal Society (London) A, Vol. 282, No. 1389, 1964, pp. 178-183.

[11] S. Chatterjee and A. Banerjee, "C-Field Cosmology in Higher Dimensions," General Relativity Gravitation, Vol. 36, No. 2, 2004, pp. 303-313.

[12] R. Bali and R. S. Tikekar, "C-Field Cosmology with Variable $G$ in the Flat Friedmann-Robertson-Walker Model," Chinese Physics Letters, Vol. 24, No. 11, 2007, pp. 3290-3292.

[13] R. Bali and M. Kumawat, "C-Field Cosmological Models with Variable G In FRW Space-Time," International Journal of Theoretical Physics, Vol. 48, No. 3, 2009, pp. 3410-3415

[14] T. Singh and R. Chaubey, "Bianchi type-I, III, V, VI and Kantowski-Sachs Universes in Creation-Field Cosmology," Astrophysical Space Scence, Vol. 321, No. 1, 2009, pp. 5-18. 